\title{
Local Targeting of Lung Tumor Associated Macrophages with Pulmonary Delivery of a CSF-1R Inhibitor for the Treatment of Breast Cancer Lung
}

\section{Metastases}

Sulaiman S. Alhudaithi ${ }^{1, \&}$, Rashed M. Almuqbil ${ }^{1} \wedge$, Hanming. Zhang ${ }^{1}$, Elizabeth R. Bielski ${ }^{1, \%}$, Wei. Du ${ }^{2}$, Fatemah S. Sunbul ${ }^{1}$, Paula D. Bos ${ }^{2,3}$, Sandro R. P. da Rocha ${ }^{1,3, *}$

${ }^{1}$ Department of Pharmaceutics and Center for Pharmaceutical Engineering and Sciences, School of Pharmacy, Virginia Commonwealth University, Richmond 23298, Virginia, United States

${ }^{2}$ Department of Pathology, School of Medicine, Virginia Commonwealth University, Richmond 23298, Virginia, United States

${ }^{3}$ VCU Massey Cancer Center, School of Medicine, Virginia Commonwealth University, Richmond 23298, Virginia, United States.

* to whom correspondence should be addressed: srdarocha@vcu.edu

\& Doctoral student, VCU Department of Pharmaceutics. Faculty on leave from the Department of Pharmaceutics, College of Pharmacy, King Saud University, Riyadh, Saudi Arabia

${ }^{\wedge}$ Doctoral student, VCU Department of Pharmaceutics. Faculty on leave from Department of Pharmaceutical Sciences, College of Clinical Pharmacy, King Faisal University, Al Ahsa, Saudi Arabia ${ }^{\%}$ Current position: Chemist in the Division of Therapeutic Performance (DTP)/ORS/OGD at FDA Orally Inhaled Drug Products 

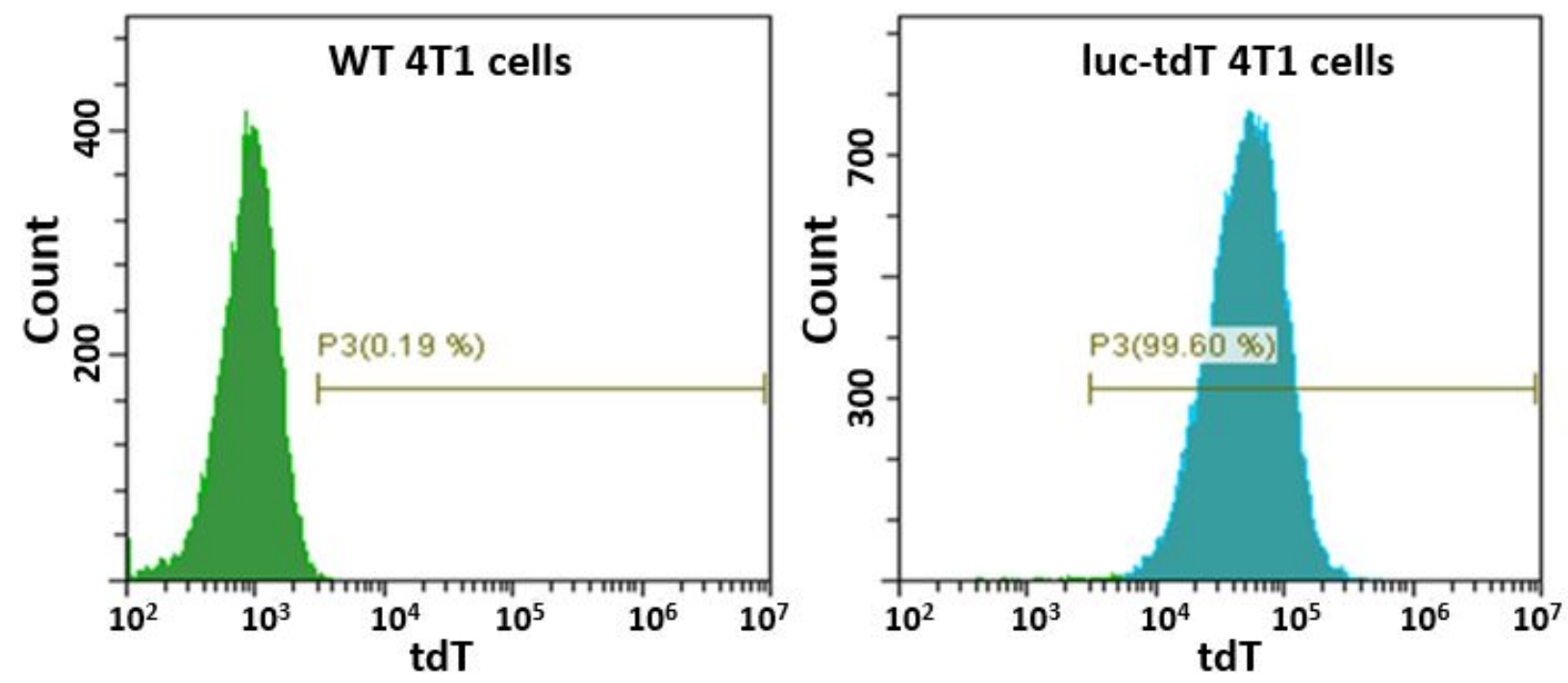

Figure S1: Flow-cytometry analysis of tdT (PE) expression on WT (left), and luc-tdT 4T1 (right), obtained after cell sorting of transduced cells. 

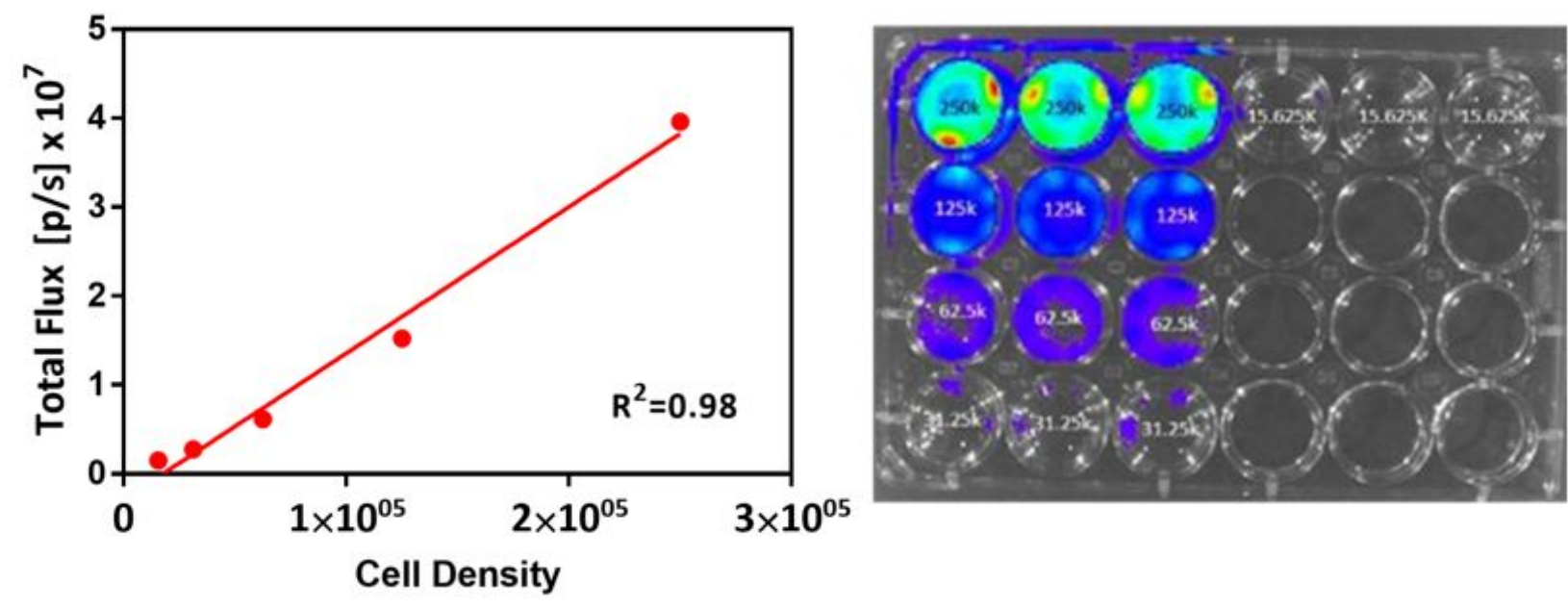

Figure S2: In vitro kinetics of luc-tdT 4T1 cells after incubation with D-luciferin for $20 \mathrm{~min}$, presented as a linear relationship between the cell density and signal intensity (left) and image of luciferase-expressing cells with various densities (right). 


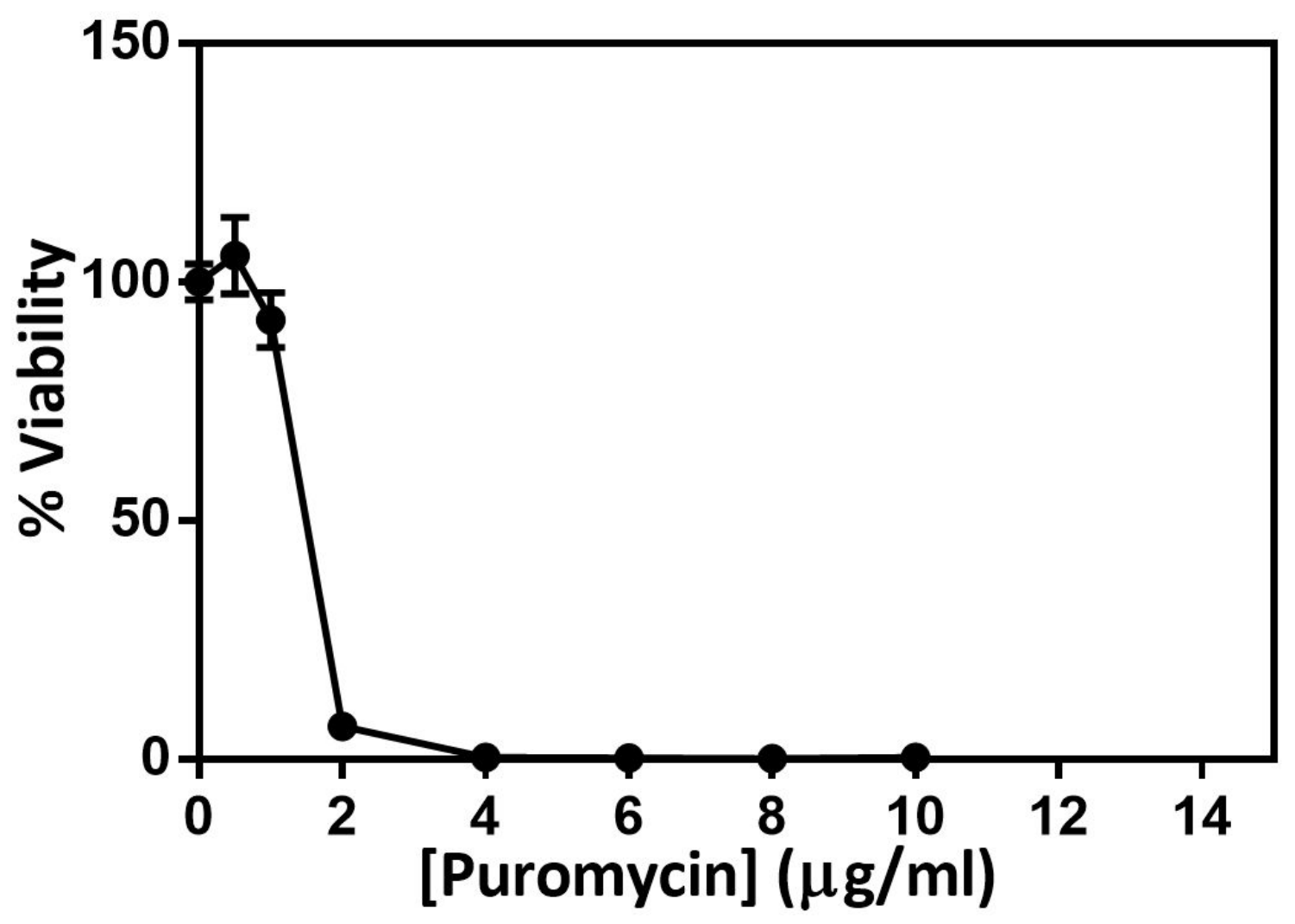

Figure S3: Puromycin cytotoxicity curve, measured by MTT assay after $48 \mathrm{~h}$ incubation with WT $4 \mathrm{~T} 1$ cells $(\mathrm{n}=6)$. Data represented as mean \pm SEM. 


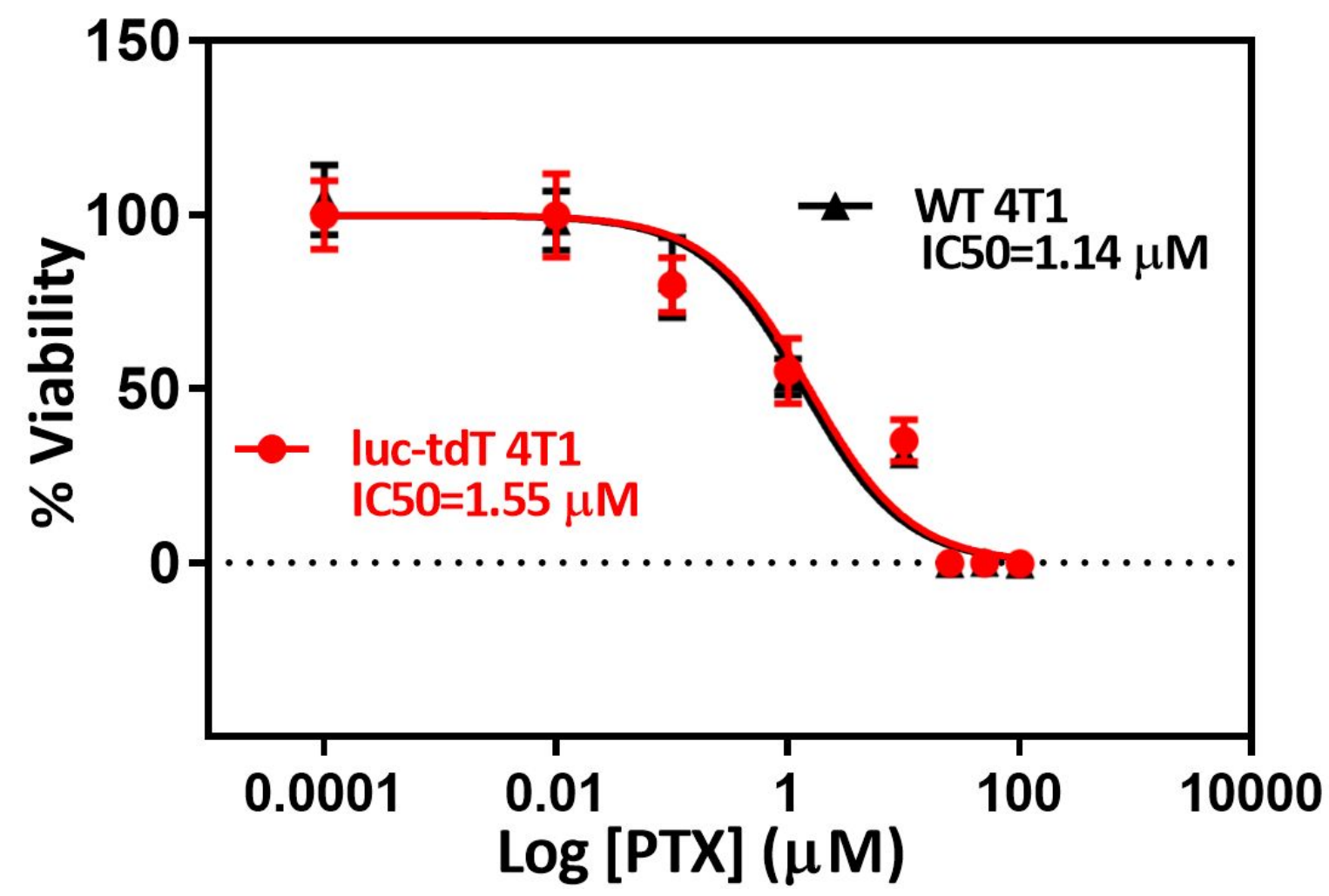

Figure S4: PTX cytotoxicity curve, measured by MTT assay after $48 \mathrm{~h}$ incubation with WT (black) and luc-tdT $4 \mathrm{~T} 1$ (red) cells $(\mathrm{n}=8) ; \mathrm{IC}_{50}$ values were determined by non-linear regression, $\log$ (inhibitor) vs normalized response. Data represented as mean $\pm \mathrm{SEM}$. 
(A)

Lung Deposition of DOX

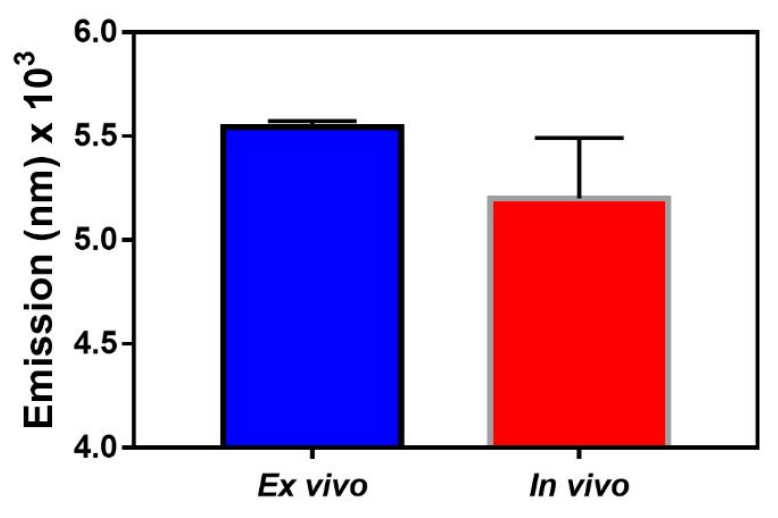

(B)

\% Lung Deposition of DOX

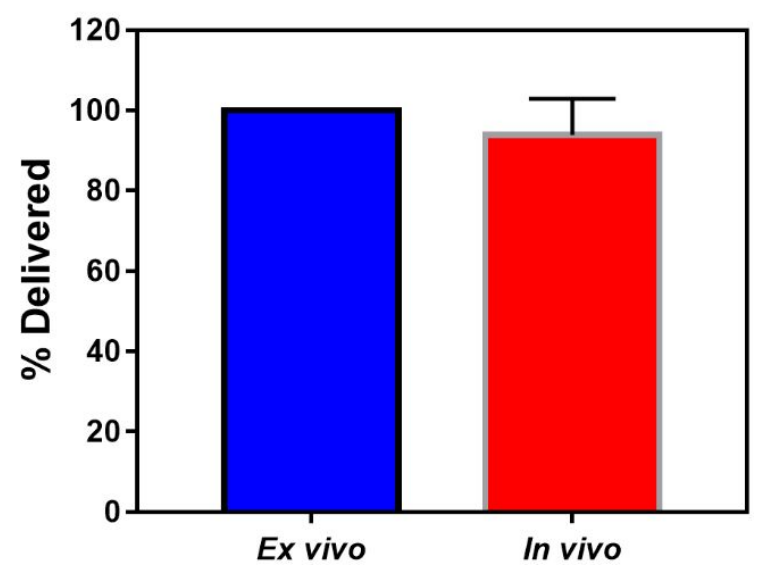

Figure S5: Assessment of lung deposition in 10-week old Balb/c animals upon pulmonary administration (endotracheal intubation) of a fluorescent dye (doxorubicin, DOX), n=3. (A) In vivo Fluorescent values are compared with ex vivo values (lungs spiked with DOX ex vivo and represent control), (B) Data are normalized to average control and represented as percentage. 

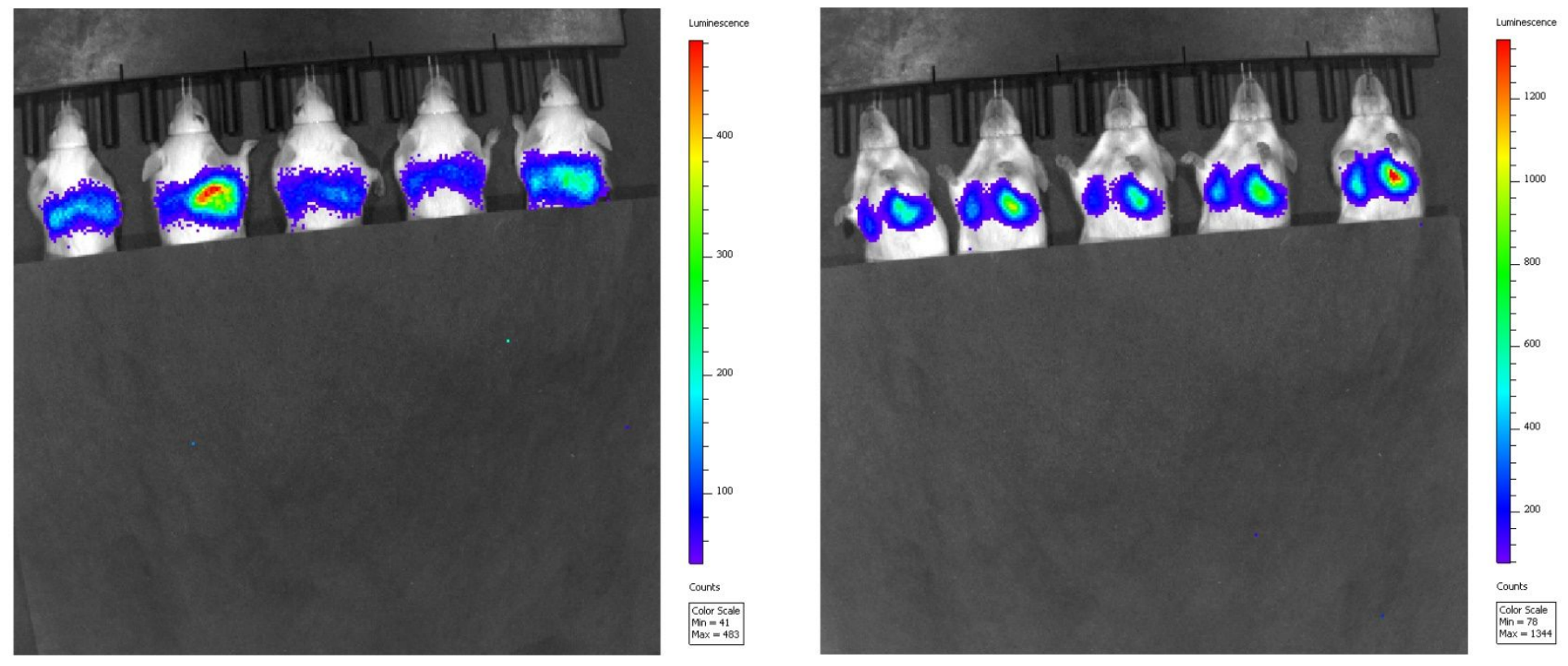

Figure S6: In vivo biolumenscince of Balb/c mice on day zero after tail vein injection of luc-tdT $4 \mathrm{~T} 1$ cells as measured by IVIS. Dorsal view (left panel) and ventral view (right panel). 


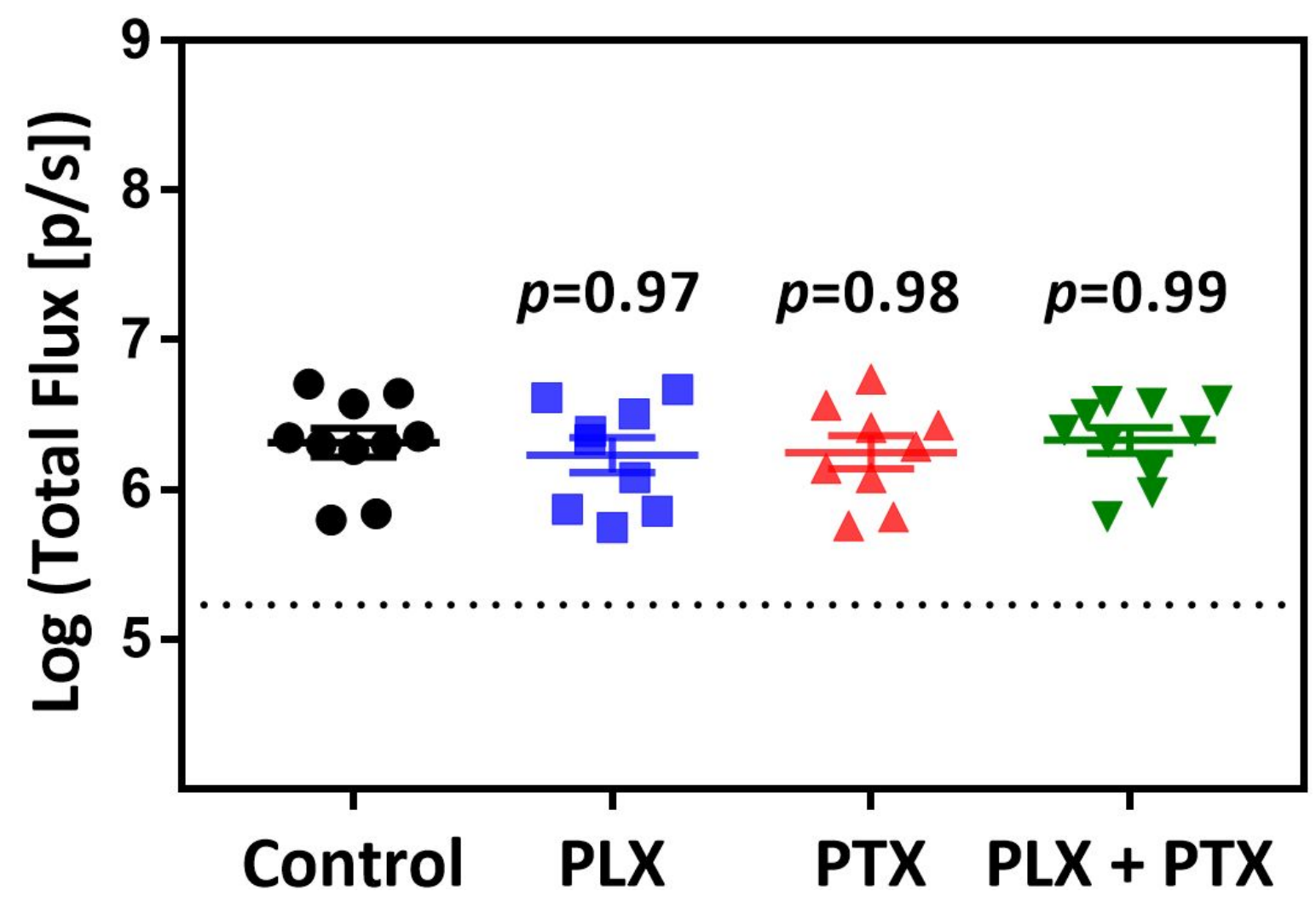




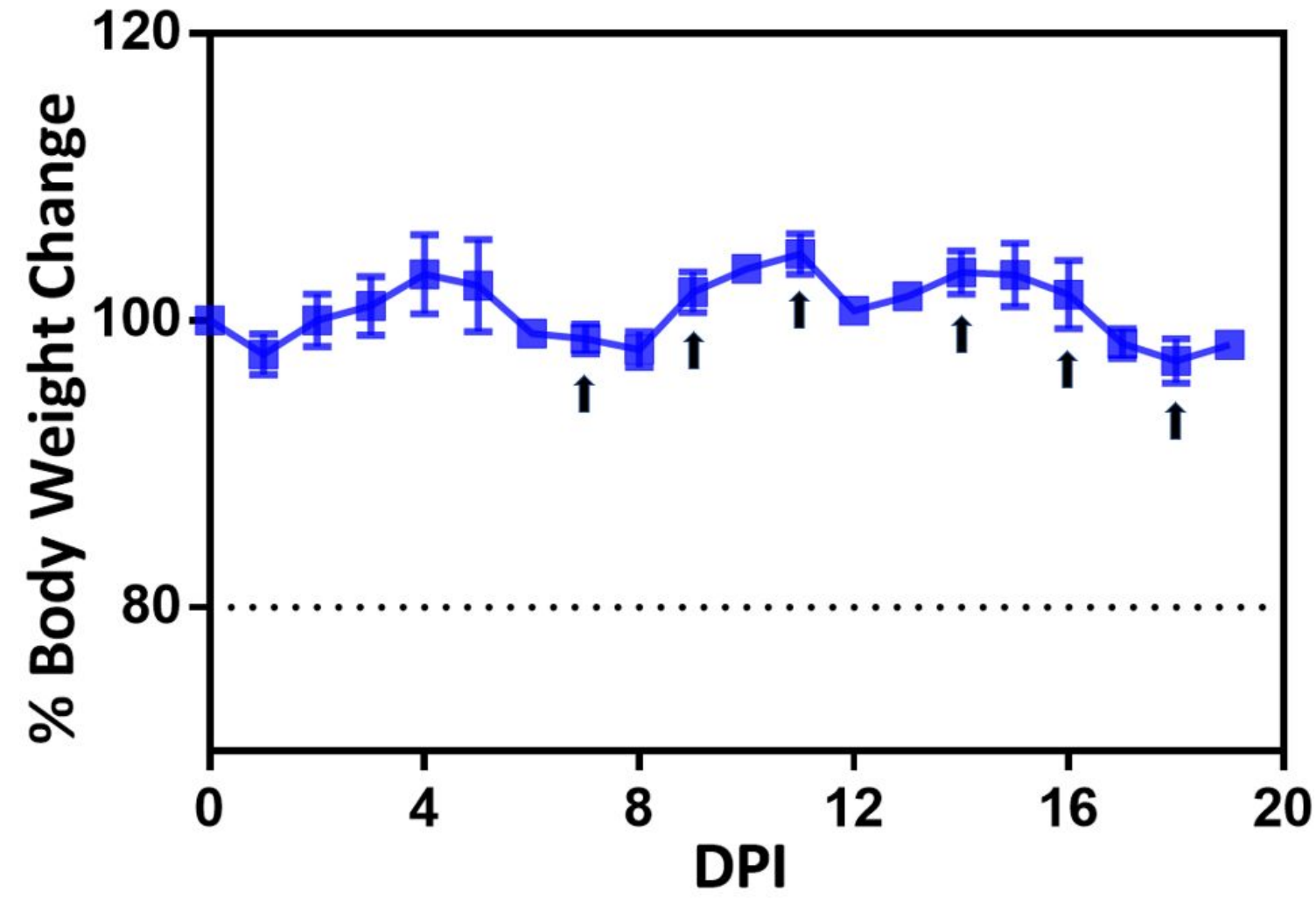

Figure S7: Total flux of treatment groups as measured by in vivo bioluminescence analysis of $\mathrm{Balb} / \mathrm{c}$ mice on day 7 after randomization and before treatment. One-way ANOVA, data represented as mean \pm SEM 
Figure S8: Measurement of body weights, represented by percentage changes from day zero $(100 \%)$ to the terminal day (day 19), of Balb/c mice treated for two cycles with $1 \times$ PBS $(n=3)$. Arrows indicate treatment days. 


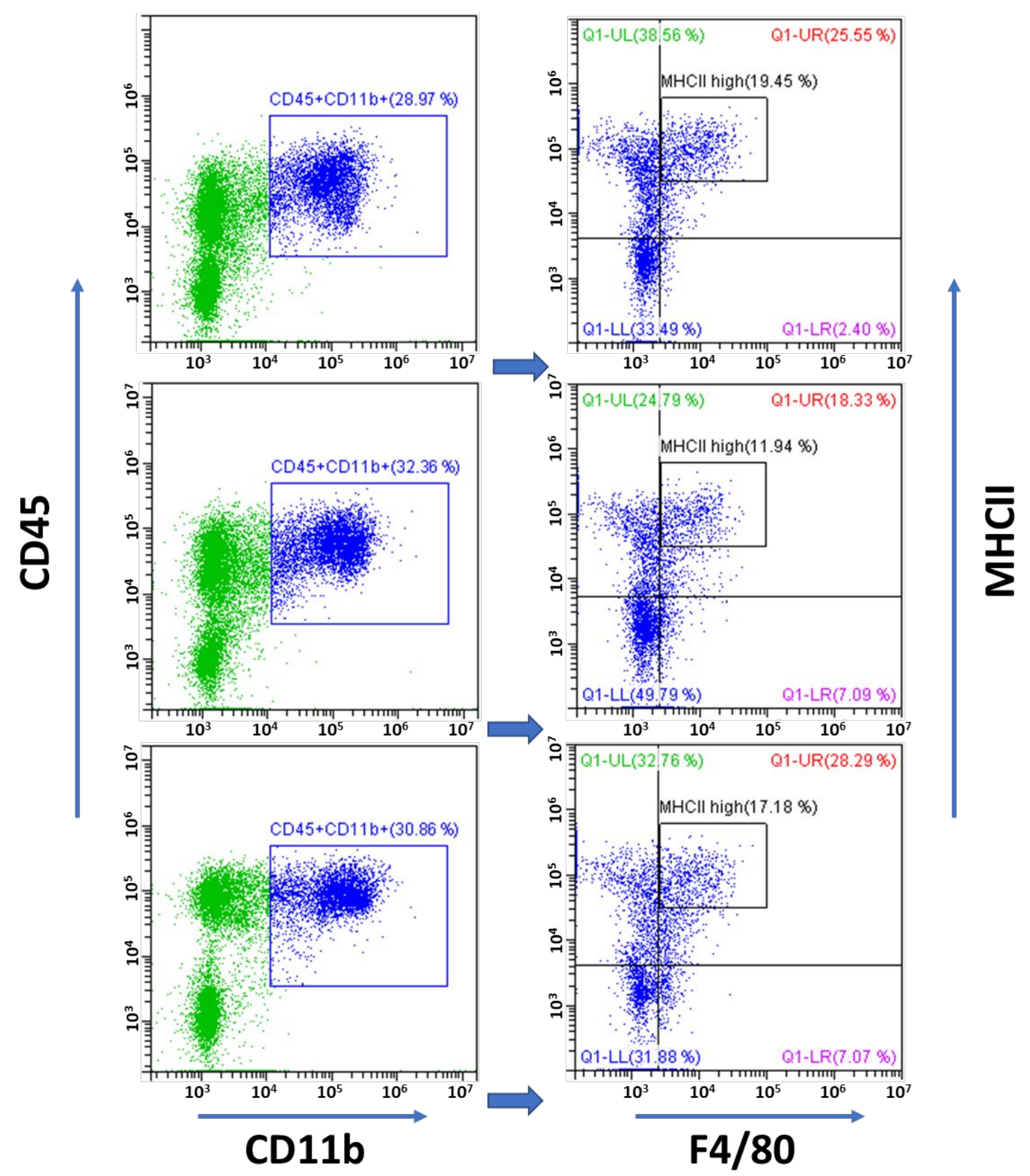

Figure S9: Gating strategy on Flow-cytometry for tumor samples (top: PLX, middle: PTX, bottom: PLX+PTX), M1 TAMs are represented by $\mathrm{CD}^{2} 5^{+} \mathrm{CD} 11 \mathrm{~b}^{+} \mathrm{F} 4 / 80^{+} \mathrm{MHCII}^{\text {high }}$ cells. 